\title{
OPEN Relationship between fatty pancreas and hypertriglyceridemic waist phenotype: a cross-sectional study
}

\author{
Xiaoping Yu $u^{1,2,3,6}$, Dan Wang ${ }^{1,4,6}$, Weiming Xiao ${ }^{1,4,6}$, Xinlin Shi ${ }^{1}$, Qiang She ${ }^{1,4}$, \\ Hongguang Sun ${ }^{3}$, Tingyue $\mathrm{Qi}^{3}$, Renyan $\mathrm{Xu}^{2,3}$, Guiqing $\mathrm{Li}^{1,4}$, Xinnong Liu ${ }^{1}$, Weijuan Gong ${ }^{1,4,5}$, \\ Zhigang Yan ${ }^{1,4}$, Yanbing Ding ${ }^{1,4} \bowtie$ \& Guotao Lu ${ }^{1,4} \bowtie$
}

Hypertriglyceridemic waist phenotype (HTWP) and its quantitative indicator, waist circumferencetriglyceride index (WTI), are common quantitative indices of visceral obesity and are closely related to metabolic diseases. The purpose of this study was to investigate the relationship between fatty pancreas (FP) and HTWP in China. FP was diagnosed using trans-abdominal ultrasonography in all participants. According to the waist circumference and serum triglyceride levels, the participants were divided into four phenotype groups: normal waist circumference-normal triglyceride, normal waist circumference-elevated triglyceride, elevated waist circumference-normal triglyceride, and elevated waist circumference-elevated triglyceride (indicating HTWP). Clinical characteristics and biochemical indices were compared among the groups. Receiver operating characteristic (ROC) curves were used to evaluate the utility of WTI as a reference factor for FP screening. The HTWP group had a higher prevalence of metabolic syndrome (84.2\%), FP (10.4\%), fatty liver (64.5\%), and hypertension (15.8\%) than the other three phenotype groups. The occurrence rate of HTWP and the median WTI were significantly higher in participants with FP than in those without FP ( $54.7 \%$ vs $21.0 \%, 222 \pm 135$ vs $142 \pm 141, p<0.001$ ). In the ROC curve analysis, when the maximum area under the curve was 0.746 , the WTI was 107.09 and the corresponding sensitivity and specificity were $90.6 \%$ and $51.9 \%$, respectively. HTWP is closely associated with FP and can be used as a reference factor for FP screening.

Obesity easily leads to the accumulation of adipose tissue in the body ${ }^{1,2}$. Central obesity, characterised by a large waist circumference (WC), is an especially obvious condition. The accumulation of adipose tissue in specific organs and tissues, such as the heart, liver, and pancreas, is referred to as "ectopic fat deposition" ${ }^{\text {. Fatty pancreas }}$ (FP) is an excessive deposition of fat in pancreatic tissue, also known as pancreatic steatosis, pancreatic fatty infiltration, or non-alcoholic FP disease ${ }^{4}$.

FP has no specific clinical manifestations and is diagnosed in most individuals on physical examination. Ultrasound and imaging studies have become the primary methods for diagnosing FP, including ultrasonography, computed tomography (CT), and magnetic resonance imaging (MRI). MRI can sensitively evaluate tissue fat content and is a good standard method for detecting pancreatic steatosis ${ }^{5}$. However, because of health economics and other factors, some new methods for the early diagnosis of FP are still warranted.

\footnotetext{
${ }^{1}$ Institute of Digestive Diseases, The Affiliated Hospital of Yangzhou University, Yangzhou University, No. 386 Hanjiang Median Road, Yangzhou 225000, Jiangsu, People's Republic of China. ${ }^{2}$ Department of Health Promotion Center, The Affiliated Hospital of Yangzhou University, Yangzhou University, Yangzhou, Jiangsu, People's Republic of China. ${ }^{3}$ Department of Ultrasound, The Affiliated Hospital of Yangzhou University, Yangzhou University, Yangzhou, Jiangsu, People's Republic of China. ${ }^{4}$ Pancreatic Center, Department of Gastroenterology, The Affiliated Hospital of Yangzhou University, Yangzhou University, Yangzhou, Jiangsu, People's Republic of China. ${ }^{5}$ Jiangsu Co-Innovation Center for Prevention and Control of Important Animal Infectious Diseases and Zoonoses, College of Veterinary Medicine, Yangzhou 225001, People's Republic of China. ${ }^{6}$ These authors contributed equally: Xiaoping Yu, Dan Wang and Weiming Xiao.『email: jsyzyzg@aliyun.com; ybding@yzu.edu.cn; gtlu@yzu.edu.cn
} 
The occurrence of FP has been reported to be associated with older age, obesity, hyperlipidaemia, fatty liver $(\mathrm{FL})^{6}$, and type 2 diabetes (T2DM). However, these findings remain controversial ${ }^{7-13}$. At present, the clinical risk factors for FP are unclear.

Lemieux et al. first proposed the concept of the hypertriglyceridemic waist phenotype (HTWP) in 2000, which was defined as elevated serum triglyceride (TG) level and elevated $\mathrm{WC}^{14}$. In practice, WC is an easily reproducible anthropometric measure, and WC measurement is the simplest method for assessing abdominal fat accumulation and the associated cardiovascular risk. In addition, WC has been widely used in recent years as a simple, low-cost index of visceral adipose tissue deposition in the abdomen ${ }^{15}$. However, WC alone cannot distinguish the extent of visceral adipose tissue and subcutaneous adipose tissue accumulation in the abdominal cavity ${ }^{16,17}$. Therefore, researchers have suggested that WC and TG level should be included as clinical indicators of visceral fat accumulation ${ }^{17}$. Substantial studies have shown that HTWP may also be used as an indicator for the early diagnosis of coronary heart disease, hypertension ${ }^{18-20}$, metabolic syndrome (MetS) $)^{21}$, and T2DM and its complications $s^{19,22-25}$ in adults ${ }^{26-30}$ and children ${ }^{21}$.

At present, no study has used HTWP to screen for FP. Therefore, our study aimed to prospectively investigate the relationship between HTWP and FP.

\section{Methods}

Ethics, consent, and permissions. This study conformed to the ethical principles of the Declaration of Helsinki. Informed consent was obtained from all participants when they underwent waist measurements, and this study was approved by the Ethics Committee of the Affiliated Hospital of Yangzhou University.

Subjects recruitments. This was a cross-sectional study. The study population, consisting of medical staff (including current and former employees) of the Affiliated Hospital of Yangzhou University, was selected in 2016, as described previously ${ }^{6}$. Briefly, the exclusion criteria were as follows: acute or chronic inflammatory disease; previous diagnosis of chronic pancreatic, liver, or kidney disease; previous diagnosis of pancreatic cancer; history of pancreatic surgery; severe immune system disorders; and pregnancy in women.

Definition of disease. WC was measured midway between the lower rib margin and the iliac crest in a standing position. Hypertriglyceridemia was defined as serum TG level $\geq 1.7 \mathrm{mmol} / \mathrm{L}$. The participants were divided into four groups according to the WC and serum TG level ${ }^{14}$ : (1) normal WC and normal TG (group1 ; WC $<90 \mathrm{~cm}$ in men and $<80 \mathrm{~cm}$ in women, TG level $<1.7 \mathrm{mmol} / \mathrm{L}$ ), (2) normal WC-elevated TG (group-2; TG level $\geq 1.7 \mathrm{mmol} / \mathrm{L}, \mathrm{WC}<80 \mathrm{~cm}$ in women and $<90 \mathrm{~cm}$ in men), (3) elevated WC-normal TG (group-3; WC $\geq 80 \mathrm{~cm}$ in women and $\geq 90 \mathrm{~cm}$ in men, TG level $<1.7 \mathrm{mmol} / \mathrm{L}$ ), and (4) elevated WC-elevated TG (HTWP group, group-4; WC $\geq 90 \mathrm{~cm}$ in men and $\geq 80 \mathrm{~cm}$ in women, blood TG level $\geq 1.7 \mathrm{mmol} / \mathrm{L})$. Additionally, the WC-TG index (WTI) was calculated as WC $(\mathrm{cm}) \times$ TG level $(\mathrm{mmol} / \mathrm{L})^{31}$.

Body mass index (BMI) $\geq 28 \mathrm{~kg} / \mathrm{m}^{2}$ was defined as obesity ${ }^{32}$. FL and FP were evaluated using trans-abdominal ultrasonography, as described previously ${ }^{6}$. A representative photograph of FP is shown in Supplementary figure S1. The diagnostic criteria for diabetes, hypertension, and MetS were based on previous studies ${ }^{33,34}$.

Statistical analysis. IBM SPSS 20.0 version software (Inc., Chicago, IL) was used for statistical analysis. Continuous variables are expressed as mean \pm standard deviation, and categorical variables are expressed as $n$ (percentage) or median (interquartile range). The basic characteristics and differences among groups were compared using analysis of variance for continuous variables. Classification variables were analysed using the $\chi^{2}$ test. A p value of $<0.05$ (two-sided) was considered statistically significant. To further investigate the relationship between HTWP and FP, we used WTI to predict FP using receiver operating characteristic (ROC) curve analysis. We calculated the best cut point according to the Youden index (= sensitivity - [1 - specificity $])$.

\section{Results}

Baseline clinical characteristics in different phenotype groups. A total of 1241 participants were included in the current study. The mean age of the participants was $45.7 \pm 14.1$ years, and $581(46.8 \%)$ were men. The participants were divided into four groups according to WC and TG level: group-1 $(n=538)$, group-2 $(n=134)$, group-3 $(n=290)$, and group-4 (HTWP group, $n=279)$. A total of $307(24.7 \%)$ participants were diagnosed with MetS. The prevalence of FP was $4.3 \%$ respectively (Table 1 ).

The baseline characteristics of the participants are shown in Tables 1 and 2. Participants with HTWP (group4) had a higher mean age (51.2 vs 41.0, 46.1, and 48.9 years), BMI (26.8 vs $21.6,23.2$, and $\left.26.0 \mathrm{~kg} / \mathrm{m}^{2}\right)$, and WC ( 93.6 vs $75.2,81.8$, and $90.2 \mathrm{~cm}$ ) than the other three phenotype groups, and group-4 also had a higher proportion of men than group- 1 and group-3 (57.3\% vs $40.9 \%$ and $35.9 \%$ ) (all $p<0.001$, Table 1 ). Moreover, individuals with HTWP had high levels of indicators related to metabolic disorder, including aspartate aminotransferase, alanine aminotransferase, gamma glutamyl transferase, serum uric acid, fasting blood glucose, TG, cholesterol, and low-density lipoprotein (all $p<0.0001$, Table 2 ). Conversely, lower levels of high-density lipoprotein were observed in the HTWP group $(p<0.0001$, Table 2$)$. In addition, the proportions of participants with smoking and alcohol drinking habits were significantly higher in group- 4 than in the other groups (all $p<0.001$, Table 1$)$.

Prevalence of FP and metabolic related diseases in the four subgroups. As shown in Table 1, group-4 had the highest prevalence of MetS (84.2\%), which showed a significant difference from group-1, group2 , and group $-3(0 \%, 20.9 \%$, and $15.2 \%$, all $p<0.001)$. Similarly, the prevalence of FP $(10.4 \%)$, FL $(64.5 \%)$, and hypertension (15.8\%) in the HTWP group were higher than those in the other three phenotype groups. The 


\begin{tabular}{|c|c|c|c|c|c|c|}
\hline & Total & G-1 & G-2 & G-3 & G-4 & $\mathrm{P}^{\mathrm{a}}$ \\
\hline $\mathrm{N}$ & 1241 & 538 & 134 & 290 & 279 & \\
\hline Male & $581 / 46.8$ & $220 / 40.9$ & $97 / 72.4^{*}$ & $104 / 35.9^{* *}$ & $160 / 57.3^{\star} \&$ & $<0.001$ \\
\hline Age & $45.7 \pm 14.1$ & $41.0 \pm 13.5$ & $46.1 \pm 12.1^{\star}$ & $48.9 \pm 14.0^{*}$ & $51.2 \pm 13.6^{* *}$ & $<0.001$ \\
\hline BMI & $23.9 \pm 3.5$ & $21.6 \pm 2.3$ & $23.2 \pm 2.2^{*}$ & $26.0 \pm 3.0^{* *}$ & $26.8 \pm 2.9^{* \pm \&}$ & $<0.001$ \\
\hline WC & $83.5 \pm 10.7$ & $75.2 \pm 6.7$ & $81.8 \pm 6.4^{*}$ & $90.2 \pm 7.9^{* *}$ & $93.6 \pm 7.3^{* \pm \&}$ & $<0.001$ \\
\hline Smoking & $247 / 19.9$ & $85 / 15.8$ & $38 / 28.4^{*}$ & $46 / 15.9^{\ddagger}$ & $78 / 28.0^{* * \& 8}$ & $<0.001$ \\
\hline Drinking & $247 / 19.9$ & $72 / 13.4$ & $45 / 33.6^{*}$ & $44 / 15.2^{\text {* }}$ & $86 / 30.8^{\star \star \&}$ & $<0.001$ \\
\hline MetS & $307 / 24.7$ & $0 / 0$ & $28 / 20.9^{*}$ & $44 / 15.2^{*}$ & $235 / 84.2^{* \neq \&}$ & $<0.001$ \\
\hline HBP & $98 / 7.9$ & $13 / 2.4$ & $8 / 6.0^{*}$ & $33 / 11.4^{*}$ & $44 / 15.8^{\star \&}$ & $<0.001$ \\
\hline T2DM & $10 / 0.8$ & $3 / 0.6$ & $0 / 0$ & $3 / 1.0$ & $4 / 1.4$ & 0.381 \\
\hline FL & $366 / 29.5$ & $43 / 8.0$ & $44 / 32.8^{*}$ & $99 / 34.1^{*}$ & $180 / 64.5^{\star \star \&}$ & $<0.001$ \\
\hline FP & $53 / 4.3$ & $3 / 0.6$ & $2 / 1.5$ & $19 / 6.6^{* *}$ & $29 / 10.4^{\star \star \& \&}$ & $<0.001$ \\
\hline
\end{tabular}

Table 1. Baseline clinical characteristics by the 4 phenotype groups. Values are expressed as means (standard deviation) or n/\% G-1 (group-1): normal waist-normal triglyceride; G-2 (group-2): normal waist-elevated triglycerides; G-3 (group-3): elevated waist-normal triglycerides; G-4 (group-4): elevated waist-elevated triglyceride (HTGW). BMI body mass index, HBP high blood pressure, MetS metabolic syndrome, T2DM type

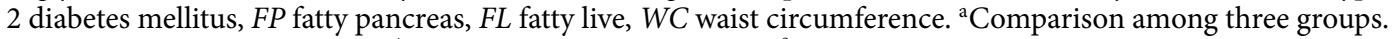
${ }^{\star}$ Compared with G-1, P $<0.05$. ${ }^{\ddagger}$ Compared with G-2, $\mathrm{P}<0.05$. ${ }^{\&}$ Compared with G-3, $\mathrm{P}<0.05$.

\begin{tabular}{|l|c|c|l|l|l|l|}
\hline & Total & G-1 & G-2 & G-3 & G-4 & P $^{\mathbf{a}}$ \\
\hline TG & $1.6 \pm 1.5$ & $1.0 \pm 0.3$ & $2.6 \pm 1.2^{*}$ & $1.2 \pm 0.3^{* *}$ & $3.1 \pm 2.2^{* \pm \&}$ & $<0.001$ \\
\hline TC & $4.4 \pm 0.9$ & $4.1 \pm 0.7$ & $4.6 \pm 1.1^{*}$ & $4.5 \pm 0.8^{*}$ & $4.7 \pm 0.9^{* \&}$ & $<0.001$ \\
\hline LDL-C & $2.5 \pm 0.7$ & $2.3 \pm 0.6$ & $2.6 \pm 0.9^{*}$ & $2.6 \pm 0.7^{*}$ & $2.6 \pm 0.7^{*}$ & $<0.001$ \\
\hline HDL-C & $1.2 \pm 0.3$ & $1.4 \pm 0.3$ & $1.1 \pm 0.3^{*}$ & $1.3 \pm 0.3^{*}$ & $1.0 \pm 0.3^{* \&}$ & $<0.001$ \\
\hline FPG & $5.2 \pm 1.2$ & $4.9 \pm 0.6$ & $5.4 \pm 1.5^{*}$ & $5.3 \pm 1.0^{*}$ & $5.7 \pm 1.7^{* \&}$ & $<0.001$ \\
\hline ALT & $25.0 \pm 19.0$ & $18.9 \pm 13.0$ & $29.8 \pm 17.5^{*}$ & $26.6 \pm 18.6^{*}$ & $32.8 \pm 25.0^{* \&}$ & $<0.001$ \\
\hline AST & $21.2 \pm 10.1$ & $18.8 \pm 7.5$ & $22.3 \pm 7.3^{*}$ & $21.5 \pm 9.5^{*}$ & $25.2 \pm 14.1^{* \pm \&}$ & $<0.001$ \\
\hline$\gamma$-GGT & $30.0 \pm 33.1$ & $18.4 \pm 15.0^{*}$ & $43.8 \pm 47.9^{*}$ & $28.8 \pm 26.9^{* *}$ & $46.5 \pm 44.4^{* \&}$ & $<0.001$ \\
\hline SCR & $74.3 \pm 33.3$ & $74.0 \pm 42.3$ & $77.1 \pm 18.4$ & $71.5 \pm 31.2$ & $76.2 \pm 15.3$ & 0.265 \\
\hline SUA & $308.4 \pm 87.5$ & $282.0 \pm 79.1$ & $333.9 \pm 84.2^{*}$ & $303.1 \pm 83.4^{* *}$ & $352.5 \pm 88.4^{* \&}$ & $<0.001$ \\
\hline
\end{tabular}

Table 2. Laboratory data by the 4 phenotype groups. Values are expressed as means (standard deviation). G-1 (group-1): normal waist-normal triglyceride; G-2 (group-2): normal waist-elevated triglycerides; G-3 (group3): elevated waist-normal triglycerides; G-4 (group-4): elevated waist- elevated triglyceride (HTGW). TG triglyceride, $T C$ total cholesterol, $L D L$ - $C$ low density lipoprotein-cholesterol, $H D L$ - $C$ high density lipoproteincholesterol, FPG fasting plasma glucose, ALT aspartate aminotransferase, AST alanine aminotransferase, $\gamma$-GGT $\gamma$-glutamyltransferase, SCR serum creatinine, SUA serum uric acid. ${ }^{a}$ Comparison among four groups. ${ }^{\star}$ Compared with G-1, P $<0.05 .{ }^{\ddagger}$ Compared with G-2, P $<0.05 .{ }^{8}$ Compared with G-3, P $<0.05 .{ }^{\#}$ Because of information incomplete, total number $=1228$.

prevalence of diabetes in the four groups gradually increased with more phenotypic abnormality; however, no statistical significance was found.

Association of the HTWP with FP. To further analyse the correlation between FP and HTWP, we divided the study population into different groups (Table 3, Fig. 1A). We quantified HTWP using the WTI. The occurrence rate of HTWP and the WTI were significantly higher in the FP group than in the non-FP group $(54.7 \%$ vs $21.0 \%, 222 \pm 135$ vs $142 \pm 141$, both $p<0.001$ ). In addition, we found that the prevalence of the elevated WCnormal TG phenotype (group-3) was higher $(\mathrm{p}<0.05)$ than that of the healthy phenotype (group-1). Moreover, no significant difference was observed between group-1 and group-2 (normal WC-elevated TG group). Furthermore, we assessed the relationship of two other metabolic diseases (FL and MetS) to WTI (Fig. 1B,C). The FL group also showed a higher proportion of participants with HTWP, higher prevalence of MetS, and higher WTI than the non-FL group $(p<0.001)$.

ROC curve: WT index predicts the FP. In the ROC curve of the WTI as a predictor of FP, the area under the curve was the largest (0.746) with a WTI of 107.09, with a corresponding sensitivity and specificity of $90.6 \%$ and $51.9 \%$, respectively (Fig. 2). 


\begin{tabular}{|c|c|c|c|c|c|c|c|c|c|}
\hline & Non-FP & FP & & Non-FL & FL & & Non-Mets & Mets & \\
\hline & $n=1188$ & $n=53$ & $\mathbf{P}$ & $\mathrm{n}=875$ & $n=366$ & $\mathbf{P}$ & $\mathrm{n}=934$ & $\mathbf{n}=\mathbf{3 0 7}$ & $\mathbf{P}$ \\
\hline G-1 & $535 / 45.1$ & $3 / 5.7$ & & $495 / 56.6$ & $43 / 11.7$ & & $538 / 57.6$ & \begin{tabular}{|l|}
$0 / 0$ \\
\end{tabular} & \\
\hline G-2 & \begin{tabular}{|l|}
$132 / 11.1$ \\
\end{tabular} & $2 / 3.8$ & $<0001$ & \begin{tabular}{|l|}
$90 / 10.3$ \\
\end{tabular} & $44 / 12.0$ & $<000$ & $106 / 11.3$ & $28 / 9.1$ & $<0001$ \\
\hline G-3 & \begin{tabular}{|l|}
$271 / 22.8$ \\
\end{tabular} & $19 / 35.8$ & $<0.001$ & \begin{tabular}{|l|}
$191 / 21.8$ \\
\end{tabular} & $99 / 27.1$ & $<0.06$ & $246 / 26.4$ & $44 / 14.3$ & $<0.001$ \\
\hline G-4 (HTGW) & \begin{tabular}{|l|l|}
$250 / 21.0$ \\
\end{tabular} & $29 / 54.7$ & & $99 / 11.3$ & $180 / 49.2$ & & $44 / 4.7$ & $235 / 76.6$ & \\
\hline WT index & $142 \pm 141$ & $222 \pm 135$ & $<0.001$ & $112 \pm 104$ & $227 \pm 179$ & $<0.001$ & $104 \pm 67$ & $274 \pm 212$ & $<0.001$ \\
\hline
\end{tabular}

Table 3. Comparison of phenotype distribution and WTI between different diseases. Values are expressed as n/\% or means (standard deviation). G-1 (group-1): normal waist-normal triglyceride; G-2 (group-2): normal waist-elevated triglycerides; G-3 (group-3): elevated waist-normal triglycerides; G-4 (group-4): elevated waistelevated triglyceride $(\mathrm{HTGW})$. WT index, waist circumference $(\mathrm{cm}) \times$ triglyceride index $(\mathrm{mmol} / \mathrm{L})$. $H B P$ high blood pressure, FP fatty pancreas, FL fatty live, Mets metabolic syndrome.

A
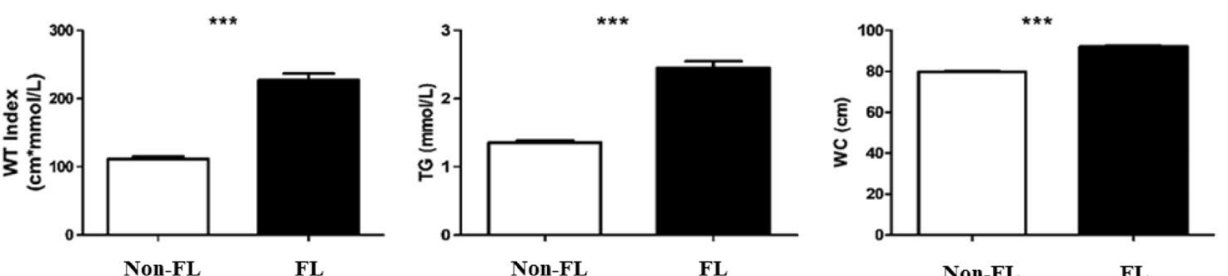

B
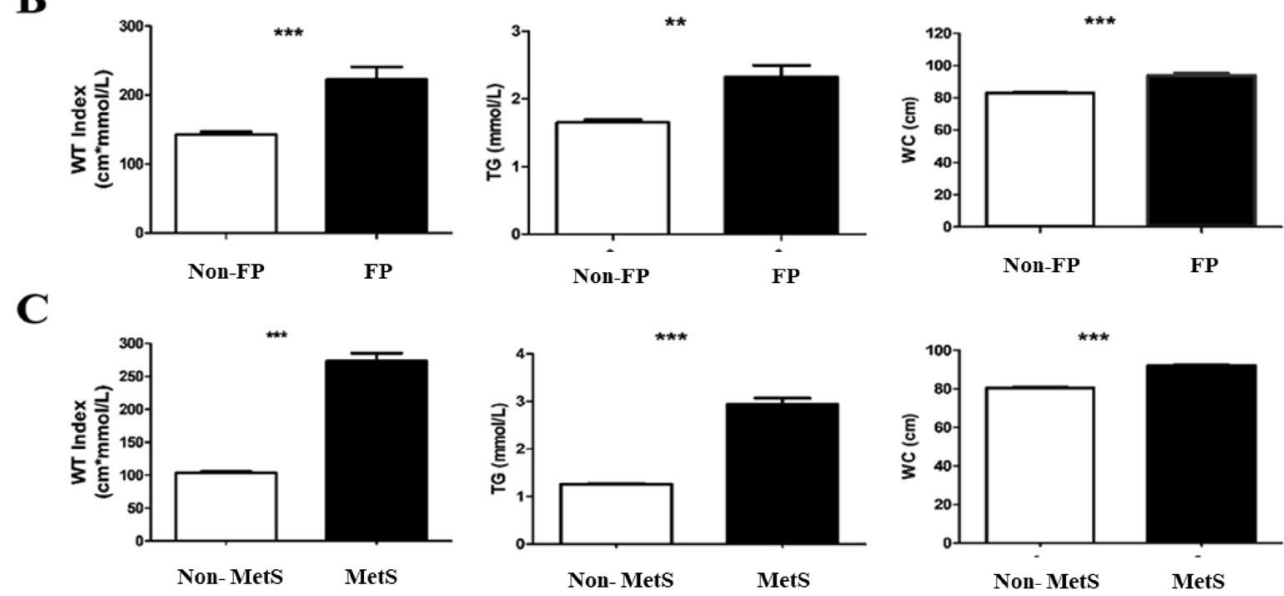

Figure 1. The histogram of WC, TG and WT index level between individuals with or without FL, FP and MetS. WC waist circumference, TG triglyceride, $W T$ index waist circumference-triglyceride index, $F P$ fatty pancreas, Non-FP non-fatty pancreas, FL fatty liver, Non-FL non-fatty liver, Mets metabolic syndrome. ${ }^{* *} \mathrm{p}<0.01$, ${ }_{* \star *} \mathrm{p}<0.001$.

\section{Discussion}

To date, no specific biomarkers for FP have been identified. Studies have demonstrated that trans-oesophageal ultrasonography, CT, and MRI are accurate methods for diagnosing $\mathrm{FP}^{35-37}$. Saisho et al. ${ }^{38}$ calculated the fat/ parenchyma ratio using CT, and the results were not significantly different from histological and anatomical findings, clearly suggesting the sensitivity of CT in the diagnosis of FP. In addition, CT and MRI have good consistency. Previous studies have reported that the location of fat infiltration is consistent in patients with FP diagnosed using CT and MRI. Currently, CT images are still commonly used to evaluate the presence of FP in the pancreas and spleen in research studies. Although CT and MRI are better methods for detecting FP, ultrasonography is the preferred large-scale screening method in daily clinical practice owing to its lower financial and time $\operatorname{costs}^{39}$. Jeong et al. demonstrated that FP can be diagnosed using trans-abdominal ultrasonography in $16.5 \%$ of cases, similar to the diagnosis rates reported in other cohort studies in the United States ${ }^{39}$, reflecting the feasibility of trans-abdominal ultrasonography.

FP is a common clinical phenomenon that is closely related to obesity, diabetes, and MetS. With increasing depth of research, clinicians' understanding of FP has gradually increased in recent years; however, there is still a large gap compared with the knowledge of FL. Additionally, studies have shown that FP is closely related to pancreatitis (acute and chronic) and pancreatic cancer, suggesting that FP may contribute to the progression of a series of pancreatic diseases. Therefore, earlier detection of FP is an urgent clinical issue. 


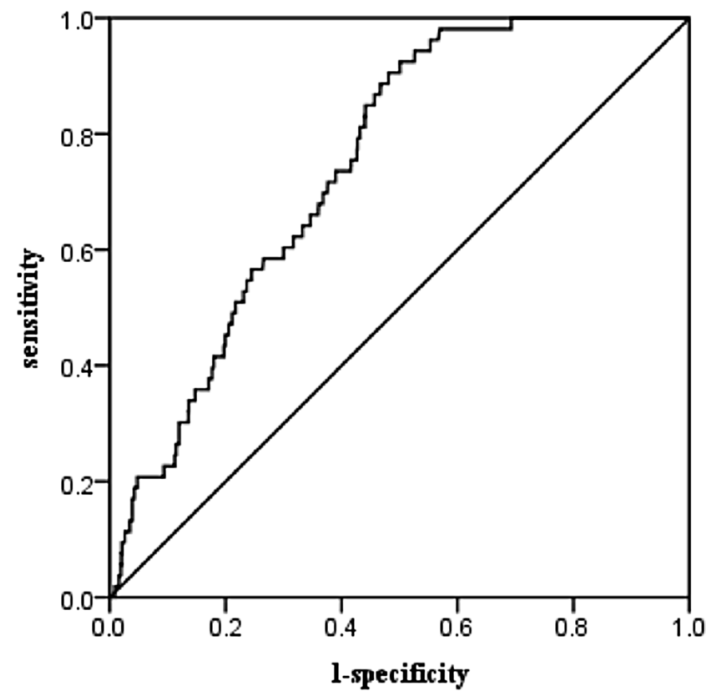

Figure 2. ROC analysis for the WT Index and FP. ROC receiver operator characteristic, WT Index waist circumference-triglyceride index.

The current study shows a clear correlation between visceral fat, waist circumference and $\mathrm{FP}^{39,40,43}$. We have previously demonstrated that only central obesity, but not general obesity, is an independent risk factor for $\mathrm{FP}^{6}$. The results suggest that a simple, easily accessible measurement of visceral obesity may have a better predictive ability for FP. HTWP, a clinical indicator of visceral fat accumulation, was first proposed by Lemieux et al. in 2000. They showed that simultaneous measurement of WC and fasting TG could be used as an inexpensive screening method to identify men at a high risk for the atherogenic metabolic triad and cardiovascular diseases ${ }^{14}$. Since then, more studies have confirmed that HTWP is closely related to the occurrence of cardiovascular diseases ${ }^{19,20}$. With further supporting research, HTWP has been considered an inexpensive and effective marker reflecting visceral (intra-abdominal) obesity and metabolic disorders. It is particularly suitable for Asians who are generally underweight but prone to the development of visceral obesity.

The correlation between HTWP and FP has not been reported. In our study, the prevalence of FP was significantly higher in the HTWP group than in the three other phenotype groups, and the proportion of participants with HTWP was relatively higher in the FP group than in the non-FP group. We also found that the prevalence of the elevated WC-normal TG phenotype (group-3) was higher $(p<0.05)$ than that of the healthy phenotype (group-1). Moreover, no significant difference was observed between group-1 and group-2 (normal WC-elevated TG group). The co-existence of elevated WC and elevated TG level had a significant additive effect on the incidence of FP. This phenomenon may be closely related to the production of free fatty acids and a pancreatic lipotoxic effect in individuals with obesity; however, the specific mechanism needs to be further clarified. In 2015, Yang et al. ${ }^{31}$ combined WC and TG into WTI for the first time, showing that WTI is a better indicator of the cardiovascular event risk in patients with coronary heart disease. Whether HTWP can be used as a risk factor for predicting FP has not been reported. In this study, we used WTI as a quantitative indicator to further predict FP. ROC curve analysis showed that the maximum area under the curve was 0.746 , and the results were statistically significant. The above results all indicated a clear correlation between HTWP and FP.

Limited clinical epidemiology studies show that the incidence rate of FP is $10-35 \%{ }^{9,11-13,41,42}$. According to a new study by Ural Koç et al. ${ }^{43}$, FP was determined visually in $29.8 \%$ of hospitalized patients (30.6\% in men and $29 \%$ in women) by non-contrast CT, in addition, the visual assessment graded of FP steatosis was almost none to mild. The incidence rate of FP in this study was $4.3 \%$, which is lower than that reported in previous studies. Clinical studies have shown that metabolic diseases and old age are independent risk factors for FP. However, in the present study, the average age of the population was $45.71 \pm 14.16$ years. The incidence rates of underlying diseases such as diabetes and hypertension were significantly lower than those in previous large epidemiological studies in China ${ }^{44}$. This may be because our study population comprised hospital staff with relatively good health. All of the above-mentioned causes eventually led to a lower incidence rate of FP in our group.

Our study had several limitations. First, all participants were health examiners from a city hospital, which resulted in a limited scope of sample collection. Second, as mentioned above, FP was diagnosed using transabdominal ultrasonography, which is not a perfect diagnostic method for this condition and may underestimate some of FP in none to mild visual assessment grade. Nevertheless, trans-abdominal ultrasonography is suitable for the health screening of large sample populations and has the advantage of being a non-invasive, fast, and economical diagnostic tool. In practice, the diagnosis and definition of FP may vary among different sonographers, which may cause bias in the data. Therefore, our ultrasound diagnosis and definition seem to be crucial. Third, our method was based on a cross-sectional study, and the causal relationship between HTWP and FP has not been determined. Fourth, the study population was obtained from a physical examination population. It was difficult to obtain accurate serological indicators of diabetes, such as serum insulin and C-peptide levels, especially in some individuals with prediabetes. Therefore, data related to the development of diabetes and 
decreased insulin secretion are lacking. To our knowledge, this is the first study to provide novel evidence of an association between HTWP and FP. More clinical studies are needed to verify our results.

\section{Conclusion}

HTWP is closely associated with FP, and WTI could be used as a preliminary screening tool for FP.

Received: 1 April 2020; Accepted: 10 November 2020

Published online: 14 December 2020

\section{References}

1. Kaplan, S. A. Re: National, Regional, and Global Trends in Body-Mass Index Since 1980: systematic analysis of health examination surveys and epidemiological studies with 960 country-years and 9.1 million participants. J. Urol. 186, 1982-1983. http://doi. org/https://doi.org/10.1016/j.juro.2011.07.061 (2011).

2. http://www.who.int/mediacentre/factsheets/fs311/en/index.html. WHO. Obesity and overweight factsheet. Updated August, 2014. Accessed November 6, 2014.

3. Despres, J. P. Inflammation and cardiovascular disease: is abdominal obesity the missing link?. Int. J. Obes. Relat. Metab. Disord. 27(Suppl 3), S22-24. https://doi.org/10.1038/sj.ijo.0802495 (2003).

4. Della Corte, C. et al. Nonalcoholic fatty pancreas disease and Nonalcoholic fatty liver disease: more than ectopic fat. Clin. Endocrinol. 83, 656-662. https://doi.org/10.1111/cen.12862 (2015).

5. Kuhn, J. P. et al. Effect of multipeak spectral modeling of fat for liver iron and fat quantification: correlation of biopsy with MR imaging results. Radiology 265, 133-142. https://doi.org/10.1148/radiol.12112520 (2012).

6. Wang, D. et al. Prevalence and clinical characteristics of fatty pancreas in Yangzhou, China: a cross-sectional study. Pancreatology 18, 263-268. https://doi.org/10.1016/j.pan.2018.02.004 (2018).

7. Hannukainen, J. C. et al. Liver and pancreatic fat content and metabolism in healthy monozygotic twins with discordant physical activity. J. Hepatol. 54, 545-552. https://doi.org/10.1016/j.jhep.2010.07.029 (2011).

8. Wu, W.-C. \& Wang, C.-Y. Association between non-alcoholic fatty pancreatic disease (nafpd) and the metabolic syndrome: casecontrol retrospective study. Cardiovasc. Diabetol. 12, 77-77. https://doi.org/10.1186/1475-2840-12-77 (2013).

9. Sepe, P. S. et al. A prospective evaluation of fatty pancreas by using EUS. Gastrointest. Endosc. 73, 987-993. https://doi.org/10.1016/j. gie.2011.01.015 (2011).

10. Lee, J. S. et al. Clinical implications of fatty pancreas: Correlations between fatty pancreas and metabolic syndrome. WJG $\mathbf{1 5}$, 1869-1875. https://doi.org/10.3748/wjg.15.1869 (2009).

11. Wang, C. Y., Ou, H. Y., Chen, M. F., Chang, T. C. \& Chang, C. J. Enigmatic ectopic fat: prevalence of nonalcoholic fatty pancreas disease and its associated factors in a Chinese population. J. Am. Heart Assoc. 3, e000297. https://doi.org/10.1161/JAHA.113.00029 7 (2014).

12. Lesmana, C. R. A., Pakasi, L. S., Inggriani, S., Aidawati, M. L. \& Lesmana, L. A. Prevalence of Non-Alcoholic Fatty Pancreas Disease (NAFPD) and its risk factors among adult medical check-up patients in a private hospital: a large cross sectional study. BMC Gastroenterol. 15, 1. https://doi.org/10.1186/s12876-015-0404-1 (2015).

13. Zhou, J. et al. The correlation between pancreatic steatosis and metabolic syndrome in a Chinese population. Pancreatology 16, 578-583. https://doi.org/10.1016/j.pan.2016.03.008 (2016).

14. Lemieux, I. et al. Hypertriglyceridemic waist: A marker of the atherogenic metabolic triad (hyperinsulinemia; hyperapolipoprotein B; small, dense LDL) in men?. Circulation 102, 179-184. https://doi.org/10.1161/01.cir.102.2.179 (2000).

15. Pouliot, M. C. et al. Waist circumference and abdominal sagittal diameter: best simple anthropometric indexes of abdominal visceral adipose tissue accumulation and related cardiovascular risk in men and women. Am. J. Cardiol. 73, 460-468. https://doi. org/10.1016/0002-9149(94)90676-9 (1994).

16. Wildman, R. P. et al. The obese without cardiometabolic risk factor clustering and the normal weight with cardiometabolic risk factor clustering: prevalence and correlates of 2 phenotypes among the US population (NHANES 1999-2004). Arch. Internal Med. 168(15), 1617-1624. https://doi.org/10.1001/archinte.168.15.1617 (2008).

17. Sam, S. et al. Hypertriglyceridemic waist phenotype predicts increased visceral fat in subjects with type 2 diabetes. Diabetes Care 32(10), 1916-1920. https://doi.org/10.2337/dc09-0412 (2009).

18. Rhee, E. J. Letter: utility of the visceral adiposity index and hypertriglyceridemic waist phenotype for predicting incident hypertension (Endocrinol Metab 2017;32:221-9, Mohsen Janghorbani et al.). Endocrinol Metab (Seoul) 32, 396-397. doi:https://doi. org/10.3803/EnM.2017.32.3.396 (2017).

19. Costa, P. R. F. et al. Hypertriglyceridemic waist phenotype and changes in the fasting glycemia and blood pressure in children and adolescents over one-year follow-up period. Arq. Bras. Cardiol. 109(1), 47-53. https://doi.org/10.5935/abc.20170067 (2017).

20. Chen, S. et al. Hypertriglyceridemic waist phenotype and metabolic abnormalities in hypertensive adults: a STROBE compliant study. Medicine 95(49), e5613. https://doi.org/10.1097/MD.0000000000005613 (2016).

21. Calcaterra, V. et al. Relation between circulating oxidized-LDL and metabolic syndrome in children with obesity: the role of hypertriglyceridemic waist phenotype. J. Pediatr. Endocrinol. Metab. 30(12), 1257-1263. https://doi.org/10.1515/jpem-2017-0239 (2017).

22. Ma, C. M. et al. The relationship between hypertriglyceridemic waist phenotype and early diabetic nephropathy in type 2 diabetes. Cardiorenal. Med. 7(4), 295-300. https://doi.org/10.1159/000477828 (2017).

23. Carlsson, A. C. Hypertriglyceridemic waist phenotype is associated with decreased insulin sensitivity and incident diabetes in elderly men. Obesity 22(2), 526-529. https://doi.org/10.1002/oby.20434 (2014).

24. Ren, Y. et al. Prevalence of hypertriglyceridemic waist and association with risk of type 2 diabetes mellitus: a meta-analysis. Diabetes Metab. Res. Rev. 32(4), 405-412. https://doi.org/10.1002/dmrr.2725 (2016).

25. Zhao, K. et al. Association between the hypertriglyceridemic waist phenotype and prediabetes in chinese adults aged 40 years and older. J. Diabetes Res. 2018, 1031939. https://doi.org/10.1155/2018/1031939 (2018).

26. Despres, J. P. et al. Abdominal obesity and the metabolic syndrome: contribution to global cardiometabolic risk. Arterioscler. Thromb. Vasc. Biol. 28, 1039-1049. https://doi.org/10.1161/atvbaha.107.159228 (2008).

27. Arsenault, B. J. et al. The hypertriglyceridemic-waist phenotype and the risk of coronary artery disease: results from the EPICNorfolk prospective population study. CMAJ 182, 1427-1432. https://doi.org/10.1503/cmaj.091276 (2010).

28. de Graaf, F. R. et al. Usefulness of hypertriglyceridemic waist phenotype in type 2 diabetes mellitus to predict the presence of coronary artery disease as assessed by computed tomographic coronary angiography. Am. J. Cardiol. 106, 1747-1753. https://doi. org/10.1016/j.amjcard.2010.08.015 (2010).

29. Cabral, N. A. et al. Hypertriglyceridemic waist and cardiometabolic risk in hypertensive women. Rev. Assoc. Med. Bras 1992(58), 568-573. https://doi.org/10.1590/s0104-42302012000500014 (2012). 
30. Wang, A. et al. Hypertriglyceridemic waist phenotype and risk of cardiovascular diseases in China: results from the Kailuan Study. Int. J. Cardiol. 174, 106-109. https://doi.org/10.1016/j.ijcard.2014.03.177 (2014).

31. Yang, R. F., Liu, X. Y., Lin, Z. \& Zhang, G. Correlation study on waist circumference-triglyceride (WT) index and coronary artery scores in patients with coronary heart disease. Eur. Rev. Med. Pharmacol. Sci. 19, 113-118 (2015).

32. Zhou, B. F. \& Cooperative Meta-Analysis Group of the Working Group on Obesity in, C. Predictive values of body mass index and waist circumference for risk factors of certain related diseases in Chinese adults: study on optimal cut-off points of body mass index and waist circumference in Chinese adults. Biomed. Environ. Sci. 15, 83-96 (2002).

33. Chobanian, A. V. et al. Seventh report of the Joint National Committee on prevention, detection, evaluation, and treatment of high blood pressure. Hypertension 42, 1206-1252. https://doi.org/10.1161/01.HYP.0000107251.49515.c2 (2003).

34. Alberti, K. G. et al. Harmonizing the metabolic syndrome: a joint interim statement of the International Diabetes Federation Task Force on Epidemiology and Prevention; National Heart, Lung, and Blood Institute; American Heart Association; World Heart Federation; International Atherosclerosis Society; and International Association for the Study of Obesity. Circulation 120, 1640-1645. https://doi.org/10.1161/CIRCULATIONAHA.109.192644 (2009).

35. Choi, C. W. et al. Associated factors for a hyperechogenic pancreas on endoscopic ultrasound. World J. Gastroenterol. 16, 4329-4334. https://doi.org/10.3748/wjg.v16.i34.4329 (2010).

36. Lingvay, I. et al. Noninvasive quantification of pancreatic fat in humans. J. Clin. Endocrinol. Metab. 94, 4070-4076. https://doi. org/10.1210/jc.2009-0584 (2009).

37. Al-Haddad, M. et al. Risk factors for hyperechogenic pancreas on endoscopic ultrasound: a case-control study. Pancreas 38, 672-675. https://doi.org/10.1097/MPA.0b013e3181a9d5af (2009).

38. Saisho, Y. et al. Pancreas volumes in humans from birth to age one hundred taking into account sex, obesity, and presence of type-2 diabetes. Clin. Anat. 20, 933-942. https://doi.org/10.1002/ca.20543 (2007).

39. Jeong, H. T., Lee, M. S. \& Kim, M. J. Quantitative analysis of pancreatic echogenicity on transabdominal sonography: correlations with metabolic syndrome. J. Clin. Ultrasound 43, 98-108. https://doi.org/10.1002/jcu.22200 (2015).

40. Singh, R. G. et al. Ectopic fat accumulation in the pancreas and its clinical relevance: a systematic review, meta-analysis, and metaregression. Metabolism 69, 1-13. https://doi.org/10.1016/j.metabol.2016.12.012 (2017).

41. Pham, Y. H. et al. Prevalence of pancreatic steatosis at a pediatric tertiary care center. S. Med. J. 109, 196-198. https://doi. org/10.14423/Smj.0000000000000432 (2016).

42. Wong, V. W. et al. Fatty pancreas, insulin resistance, and beta-cell function: a population study using fat-water magnetic resonance imaging. Am. J. Gastroenterol. 109, 589-597. https://doi.org/10.1038/ajg.2014.1 (2014).

43. Ural Koç, O. T. Evaluation of pancreatic steatosis prevalence and anthropometric measurements using non-contrast computed tomography. Turk. J. Gastroenterol. 31, 640-648. https://doi.org/10.5152/tjg.2020.19434 (2020).

44. Lu, J. et al. Prevalence, awareness, treatment, and control of hypertension in China: data from 1.7 million adults in a population-based screening study (China PEACE Million Persons Project). Lancet 390, 2549-2558. doi:https://doi.org/10.1016/S0140 $-6736(17) 32478-9$ (2017).

\section{Acknowledgements}

This work was supported by the National Natural Science Foundation of Yangzhou City (SQN20140063) and the Postdoctoral Science Foundation of China (2014M562664). We thank our colleagues, Zhang M, Zhu QT, Jiao $\mathrm{XP}, \mathrm{Wu}$ J, Teng DL, and $\mathrm{Wu} \mathrm{KY}$, for their help.

\section{Author contributions}

G.T.L., Y.B.D., and Z.G.Y. designed the study. X.P.Y., T.Y.Q., H.G.S., and G.Q.L. were responsible for the diagnosis of FP. X.P.Y., D.W., X.L.S., R.Y.X., and Q.S. were responsible for data collection and data analysis. D.W. and X.L.S. were responsible for manuscript drafting. W.M.X., X.N.L., and W.J.G. reviewed the manuscript.

\section{Competing interests}

The authors declare no competing interests.

\section{Additional information}

Supplementary Information The online version contains supplementary material available at https://doi. org/10.1038/s41598-020-78883-1.

Correspondence and requests for materials should be addressed to Z.Y., Y.D. or G.L.

Reprints and permissions information is available at www.nature.com/reprints.

Publisher's note Springer Nature remains neutral with regard to jurisdictional claims in published maps and institutional affiliations.

Open Access This article is licensed under a Creative Commons Attribution 4.0 International License, which permits use, sharing, adaptation, distribution and reproduction in any medium or format, as long as you give appropriate credit to the original author(s) and the source, provide a link to the Creative Commons licence, and indicate if changes were made. The images or other third party material in this article are included in the article's Creative Commons licence, unless indicated otherwise in a credit line to the material. If material is not included in the article's Creative Commons licence and your intended use is not permitted by statutory regulation or exceeds the permitted use, you will need to obtain permission directly from the copyright holder. To view a copy of this licence, visit http://creativecommons.org/licenses/by/4.0/.

(C) The Author(s) 2020 